\title{
Resonance modes of plasmonic nanorod metamaterials and their applications
}

Zhang, Junxi, Hu, Zhijia, Xie, Kang, Wei, Yongqin, Wang, Haoyu, et al.

Junxi Zhang, Zhijia Hu, Kang Xie, Yongqin Wei, Haoyu Wang, Fei Wang, Taiming Liang, Sonia Ghafoor, Pei Wang, Guangtao Fei, Lide Zhang, Lin Zhang, "Resonance modes of plasmonic nanorod metamaterials and their applications," Proc. SPIE 11336, AOPC 2019: Nanophotonics, 113360P (18 December 2019); doi: 10.1117/12.2547790

SPIE. Event: Applied Optics and Photonics China (AOPC2019), 2019, Beijing, China 


\title{
Resonance modes of plasmonic nanorod metamaterials and their application
}

\author{
Junxi Zhang ${ }^{1,2, a *}$, Zhijia Hu ${ }^{1,2,3}$, Kang Xie ${ }^{1, b}{ }^{*}$, Yongqin Wei ${ }^{1}$, Haoyu Wang ${ }^{1}$, Fei Wang ${ }^{1}$, Taiming \\ Liang $^{1}$, Sonia Ghafoor ${ }^{4}$, Pei Wang ${ }^{4}$, Guangtao $\mathrm{Fei}^{5}$, Lide Zhang ${ }^{5}$ and Lin Zhang ${ }^{2}$ \\ ${ }^{1}$ Department of Opto-electronic Information Science and Engineering, School of Instrument Science \\ and Opto-electronics Engineering, National Engineering Lab of Special Display Technology, Key \\ Lab of Advanced Display Technology, Hefei University of Technology, Hefei 230009, China \\ ${ }^{2}$ Aston Institute of Photonic Technologies, School of Engineering \& Applied Science, Aston \\ University, Birmingham B4 7ET, UK \\ ${ }^{3}$ State Key Laboratory of Environment-Friendly Energy Materials, Southwest University of Science \\ and Technology, Mianyang 621000, China \\ ${ }^{4}$ Department of Optics and Optical Engineering, Anhui Key Laboratory of Optoelectronic Science \\ and Technology, University of Science and Technology of China, Hefei 230026, China \\ ${ }^{5}$ Key Laboratory of Materials Physics and Anhui Key Laboratory of Nanomaterials and \\ Nanostructures, Institute of Solid State Physics, Chinese Academy of Sciences, Hefei 230031, China \\ aemail: junxi.zhang@hfut.edu.cn, bemail: kangxie@hfut.edu.cn
}

\begin{abstract}
Plasmonic nanorod metamaterials exhibit transversal and longitudinal resonance modes. It is found that the resonance intensity of the transversal modes (T-Modes) excited by the $p$-polarized wave is obviously larger than the intensity for the $s$ - polarized wave at the wavelength of the transversal resonance, and the resonance intensity of the longitudinal modes (L-Modes) excited by the $s$ - polarized wave is clearly larger than the intensity for the $p$-polarized wave at the longitudinal resonance wavelength, indicating a distinct polarization characteristics, which results from excitation of the different resonance modes of surface plasmons at different wavelengths. Moreover, the polarization behavior in near field regions for the different resonance modes has been demonstrated by the electric field distributions of the plasmonic nanorods based on FDTD simulation. In addition, the working wavelength of the polarizer can be tuned by the diameter and length of the silver nanorods in the visible spectral range, higher extinction ratios and lower insertion losses can be achieved based on the different resonance modes associated with the different polarizations. The polarizers will be a promising candidate for its potential applications in integration of nanophotonic devices.
\end{abstract}

Keywords: Plasmonic nanorod metamaterial, Resonance mode, T-Mode, L-Mode, Polarizer

\section{INTRODUCTION}

Plasmonic resonant cavities are capable of confining light at the nanometer scale due to both enhanced local electromagnetic fields and lower mode volumes, which exhibit promising applications in sources ${ }^{1-3}$, sensing ${ }^{4-6}$, enhanced effects ${ }^{7}$ etc. It has been noted that the conventional metal nanostrips as cavities with single metal-dielectric interfaces have large Ohmic losses ${ }^{8-11}$. Whereas enhancement of plasmonic near-field coupling by designing two or multi metal-dielectric interfaces can decrease the Ohmic losses ${ }^{12-15}$. In this case, the plasmonic resonant cavities with the two or multi metal-dielectric interfaces can be expected to be applied in some optical devices. Polarizers enable control the polarization state of light, are one of the most fundamental elements used in the optical devices. In this paper, we report the plasmonic resonant for their application in the polarizers.

AOPC 2019: Nanophotonics, edited by Zhiping Zhou, Xiaocong Yuan,

Daoxin Dai, Proc. of SPIE Vol. 11336, 113360P - @ 2019 SPIE

CCC code: $0277-786 X / 19 / \$ 21 \cdot$ doi: $10.1117 / 12.2547790$ 


\section{METHOD}

The plasmonic properties of the silver nanorod metamaterials can be simulated by a finite-difference time-domain (FDTD) method with Lumerical package. The refractive index of silver is based on data from Palik $(0-2 \mu \mathrm{m})$ in the material library of the FDTD software, the simulation domain covers a silver nanorod which has an arrangement of hexegonal lattice in $(\mathrm{x}, \mathrm{y})$ plane, and the mesh accuracy is 6 , the boundary conditions are the perfectly matched layer (PML) boundary conditions in the direction of $\mathrm{z}$ and the bloch boundary conditions in the $\mathrm{x}$ and $\mathrm{y}$ directions. The plane waves ( $s$ - and/or $p$ - polarized light) as incident sources with the wavelengths covering from $300 \mathrm{~nm}$ to 1000 $\mathrm{nm}$ are illuminated onto the cross-sectional surface of the silver nanorod metamaterial.

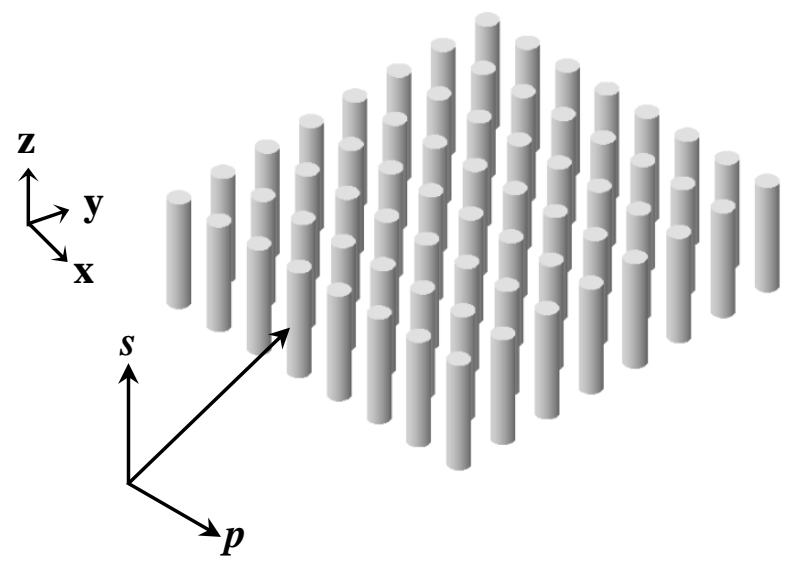

Figure 1. Schematic illustration of a silver nanorod metamaterial when the incident light with $s$ and $p$ polarization directions impinges on the cross-sectional surface of the nanorods at normal incidence along y direction, where $\mathrm{z}$ direction is parallel to the long axis of the nanorods.

\section{RESULTS AND DISCUSSION}

Near-field mode coupling of the plasmonic nanorod metamaterials consisting of the silver nanorod arrays has been investigated in our previous work ${ }^{13}$. Reflectance measurements reveal the existence of multiple resonance modes of the nanorod metamaterials. Furthermore, FDTD numerical simulations display that the electromagnetic fields of the longitudinal resonance modes are focused at the interface between silver nanorods and aluminum oxide, and the electric fields at the interface form standing waves, indicating that the nanorod metamaterials can work as plasmonic resonant cavities. Also, the resonance modes of the resonant cavities can be tuned by changing the structure parameters of the nanorod metamaterials. Our previous investigations demonstrated the high-order harmonic modes of the longitudinal resonances are observed when the nanorod length increased to $280 \mathrm{~nm}$.

In terms of the plasmonic nanorod metamaterials for application in the polarizers, we only consider the lower-order harmonic modes of the longitudinal resonances. Figure 2 shows the spectra of localized surface plasmons of the silver nanorod metamaterials with the length of $150 \mathrm{~nm}$ and the diameter of $60 \mathrm{~nm}$. The transversal resonance mode (T-Mode) at $523 \mathrm{~nm}$ can be excited by the $p$ - polarized wave (e.g., the polarization direction is along the short axis of the silver nanorods), while the longitudinal resonance mode (L-Mode) at $704 \mathrm{~nm}$ can be excited by the $s$ - polarized wave (e.g., the polarization direction is along the long axis of the nanorods). Moreover, it is found that the resonance intensity of the transversal mode for the $p$ - polarized wave is obviously larger than the intensity for the $s$ - polarized wave at the same wavelength of $523 \mathrm{~nm}$. Also, the resonance intensity of the longitudinal mode for the $s$ - polarized wave is clearly larger than the intensity for the $p$ - polarized wave at the same wavelength of $704 \mathrm{~nm}$. These results indicate that the silver nanorod metamaterials attenuate the incident light at the resonance wavelength (e.g., $523 \mathrm{~nm}$ or $704 \mathrm{~nm}$ ) of one resonance mode (e.g., T-Mode or L-Mode) for one polarized wave (e.g., $p$ - polarization or $s$ - polarization), while, they nearly completely transmit the incident light at the same wavelength for the other polarized wave ( $s$ - polarization or $p$ polarization), which exhibits distinct polarization characteristics. 


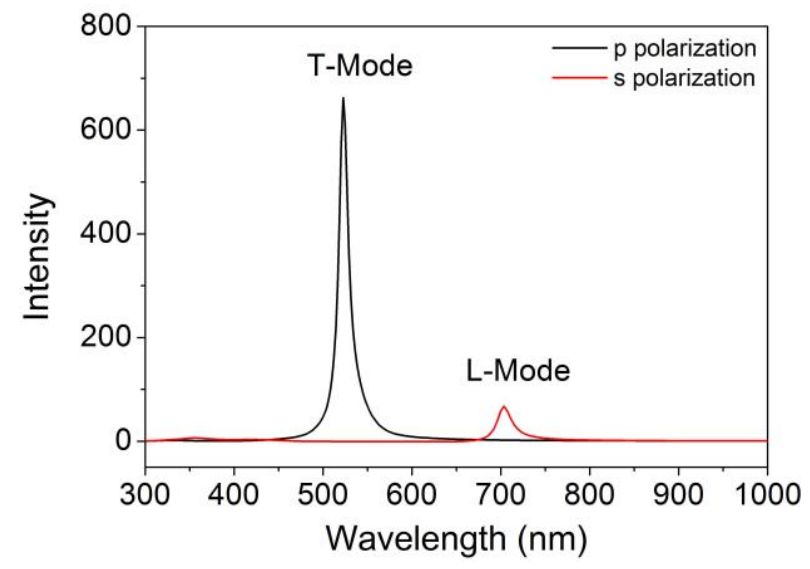

Figure 2. The spectra of localized surface plasmons of the silver nanorod metamaterials with the length of $150 \mathrm{~nm}$ and the diameter of $60 \mathrm{~nm}$ for $p$ - and $s$ - polarized waves. Where, the T-Mode is excited by the $p$ - polarized wave (black curve) and the L-Mode is excited by the $s$ - polarized wave (red curve).

Moreover, the polarization behavior in near field regions can be demonstrated by the electric field distributions of the plasmonic nanorod metamaterials based on FDTD simulation. It is observed that the resonance direction of the T-mode at $523 \mathrm{~nm}$ is along the short axis of the nanorods (i.e. x direction) based on the excitation by the $p$ - polarization of incident light, and the electric field in the near filed at $523 \mathrm{~nm}$ is very strong due to their near filed coupling of the TMode between the adjacent silver nanorods (Figure 3a). Whereas, the resonance direction at $523 \mathrm{~nm}$ is along the long axis of the nanorods (i.e. $\mathrm{z}$ direction) for the $s$ - polarization of incident light, and the electric field in the near filed at 523 $\mathrm{nm}$ is very weak because the near filed coupling of the L-Mode between the adjacent silver nanorods does not occur for the same wavelength of $523 \mathrm{~nm}$ (Figure 3b). On the other hand, the resonance direction of the L-mode at $704 \mathrm{~nm}$ is along the long axis of the nanorods (i.e. $\mathrm{z}$ direction) based on the excitation by the $s$ - polarized wave, and the electric field in the near filed at $704 \mathrm{~nm}$ is very strong due to their near filed coupling of the L-Mode between the adjacent silver nanorods (Figure 3c). Nevertheless, the resonance direction at $704 \mathrm{~nm}$ is along the short axis of the nanorods (i.e. $x$ direction) for the $p$ - polarized wave, and the electric field in the near filed at $704 \mathrm{~nm}$ is very weak because the near filed coupling of the T-Mode between the adjacent silver nanorods does not occur for the same wavelength of $704 \mathrm{~nm}$ (Figure 3d). Consequently, the plasmonic nanorod metamaterial exhibits an obvious near-field polarization characteristic at the same wavelengths associated with the two different resonance modes (T-Mode or L-Mode) excited by $p$ - and $s$ polarized waves. 

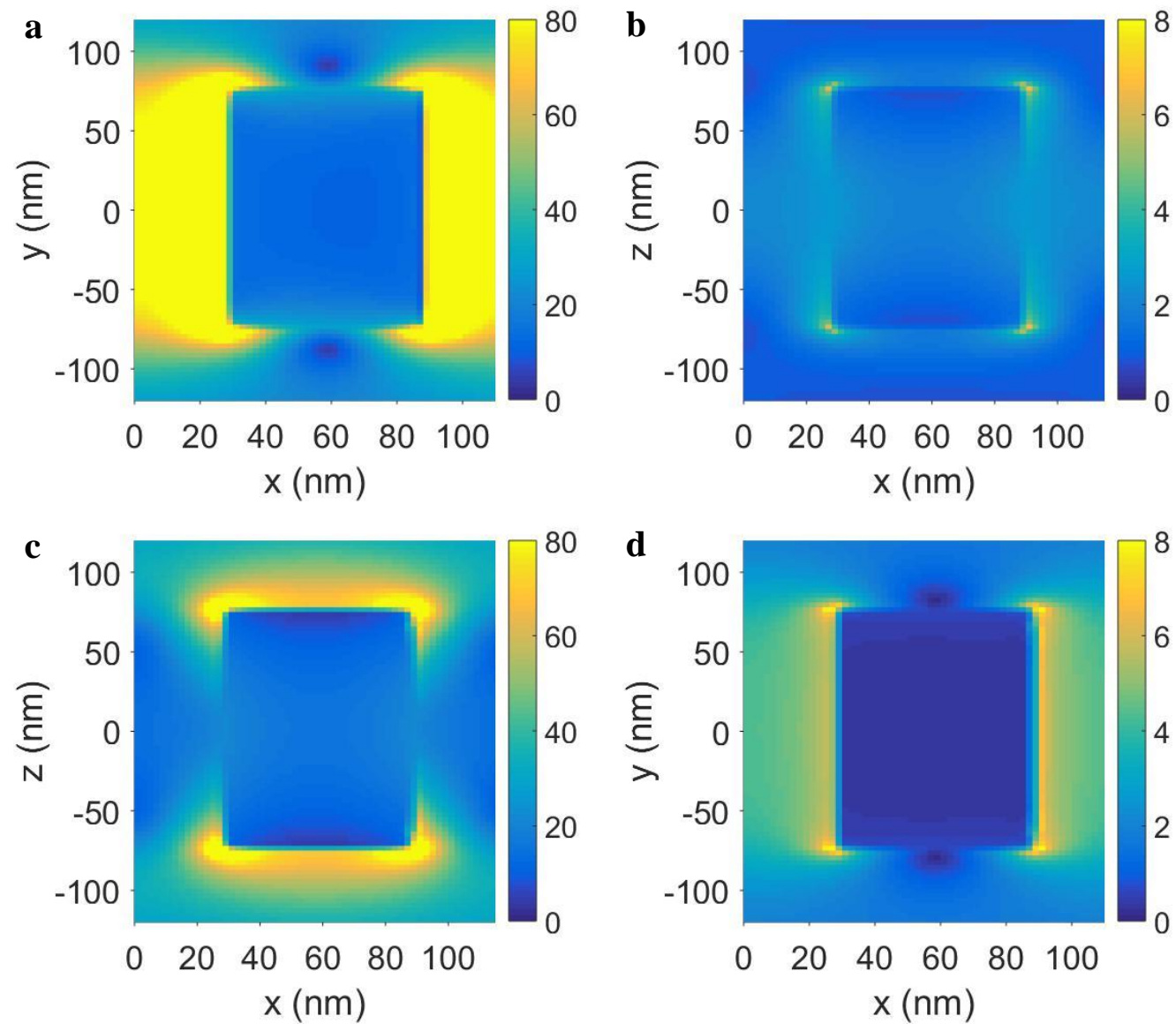

Figure 3. The electric field distributions of the silver nanorod metamaterials with the length of $150 \mathrm{~nm}$ and the diameter of $60 \mathrm{~nm}$. (a) The electric field profiles of $\mathrm{E}(\mathrm{x}, \mathrm{y})$ at $523 \mathrm{~nm}$ for the T-Mode excited by the $p$ - polarized wave; (b) The electric field profiles of $\mathrm{E}(\mathrm{x}, \mathrm{z})$ at $523 \mathrm{~nm}$ for the $s$ - polarized wave; (c) The electric field profiles of $\mathrm{E}(\mathrm{x}, \mathrm{z})$ at $704 \mathrm{~nm}$ for the L-Mode excited by the $s$ - polarized wave; (d) The electric field profiles of $\mathrm{E}(\mathrm{x}, \mathrm{y})$ at $704 \mathrm{~nm}$ for the $p$ - polarized wave.

In addition, the working wavelength of the polarizers can be tuned by changing the sizes of the silver nanorod metamaterials. Figure 4 displays the spectra of localized surface plasmons of the silver nanorod metamaterials with the nanorod diameter when the nanorod length is fixed. The T-Mode exhibits obvious redshifts from $494 \mathrm{~nm}$ to $523 \mathrm{~nm}, 564$ $\mathrm{nm}$ and $604 \mathrm{~nm}$ when the diameter of the silver nanorods increases from $50 \mathrm{~nm}$ to $60 \mathrm{~nm}, 70 \mathrm{~nm}$ and $80 \mathrm{~nm}$ for the $p$ polarized wave. In contrast, the resonance wavelength of the L-Mode doses not present clear shifts. Thus, the wavelength of the polarizers based on the T-Modes can be tailored in a wide wavelength range by adjusting the diameter of the silver nanorods. Furthermore, the resonance of the T-Modes excited by the $p$ - polarized wave are very strong than that of the LModes excited by the $s$ - polarized wave, so the higher extinction ratios and the lower insertion losses can be obtained by the resonance of the T-Modes excited by the $p$ - polarized wave and the out of the resonance by the $s$ - polarized wave at the same wavelengths. 

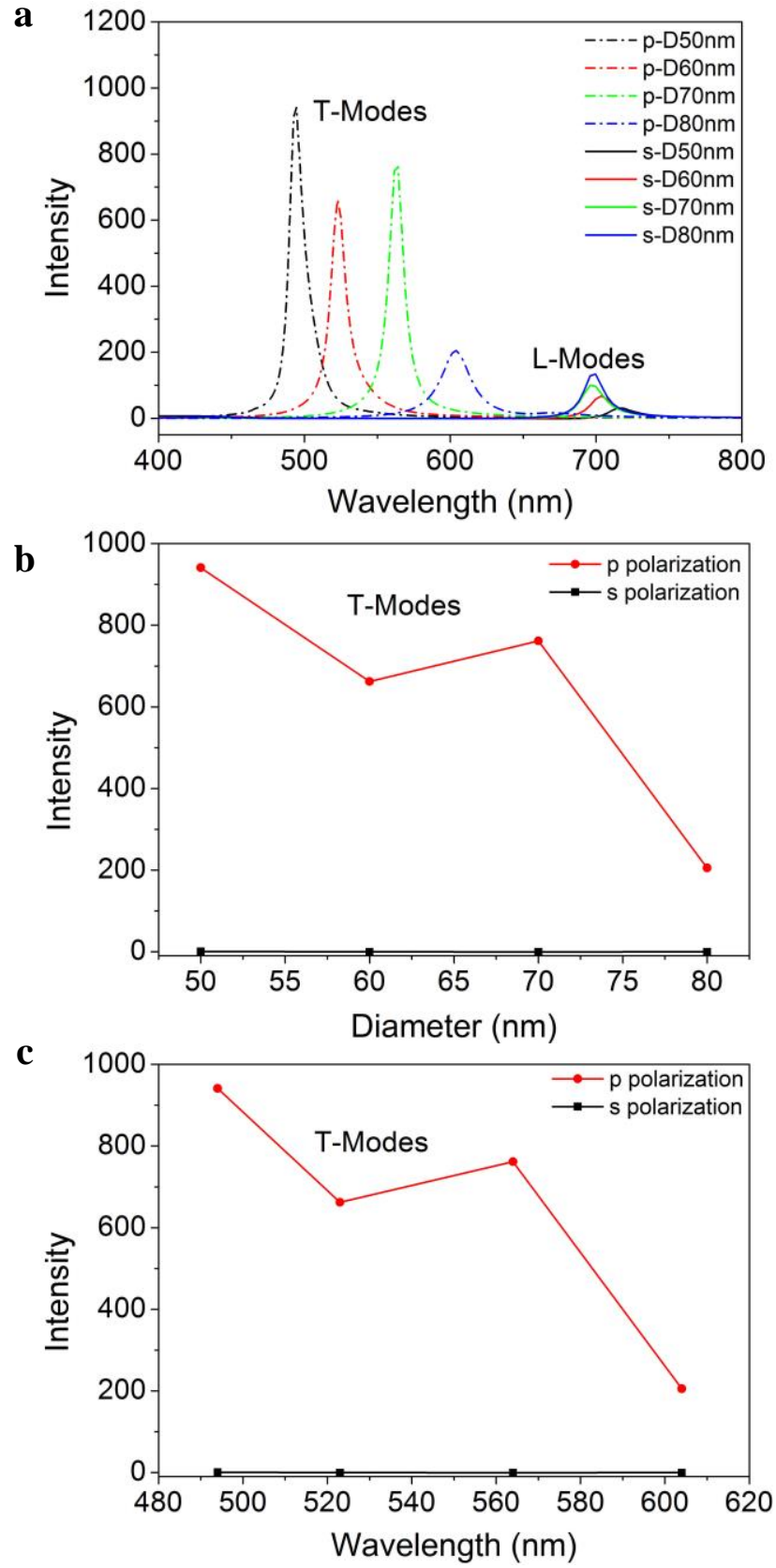

Figure 4. The spectra of localized surface plasmons of the silver nanorod metamaterials with the nanorod diameters of $50 \mathrm{~nm}$, $60 \mathrm{~nm}$, $70 \mathrm{~nm}$ and $80 \mathrm{~nm}$ for the same nanorod length of $150 \mathrm{~nm}$. (a) T-Modes excited by the $p$-polarized wave (dash dot curves) and LModes excited by the s- polarized wave (solid curves); (b) T-Modes excited by the $p$ - polarized wave for the different diameters of the nanorods (red curve), the resonance at the T-Mode wavelengths by the $s$ - polarized wave for the different diameters (black curve); (c) T-Modes excited by the $p$ - polarized wave for the different wavelengths corresponding to the different diameters of the nanorods (red curve), the out of resonance at the T-Mode wavelengths by the $s$ - polarized wave for the different wavelengths (black curve). 
Figure 5 shows the spectra of localized surface plasmons of the silver nanorod metamaterials with the nanorod length when the nanorod diameter is constant. The L-Mode displays remarkable redshifts from $559 \mathrm{~nm}$ to $630 \mathrm{~nm}, 703 \mathrm{~nm}$ and $785 \mathrm{~nm}$ when the length of the silver nanorods increases from $90 \mathrm{~nm}$ to $120 \mathrm{~nm}, 150 \mathrm{~nm}$ and $180 \mathrm{~nm}$ for the $s$ - polarized wave. In contrast, the shift of the resonance wavelength of the T-Mode is not obvious. Aa a result, the wavelength of the polarizers based on the L-Modes can be modulated in a broad waveband range by changing the length of the silver nanorods. Since the resonance of the T-Modes excited by the $p$ - polarized wave are very strong than that of the L-Modes excited by the $s$ - polarized wave, the resonance wavelength of the T-Mode has a slight shift. In these cases, the higher extinction ratios and the lower insertion losses can be obtained by the resonance of the T-Modes excited by the $p$ polarized wave and the out of the resonance by the $s$ - polarized wave at the same wavelengths.
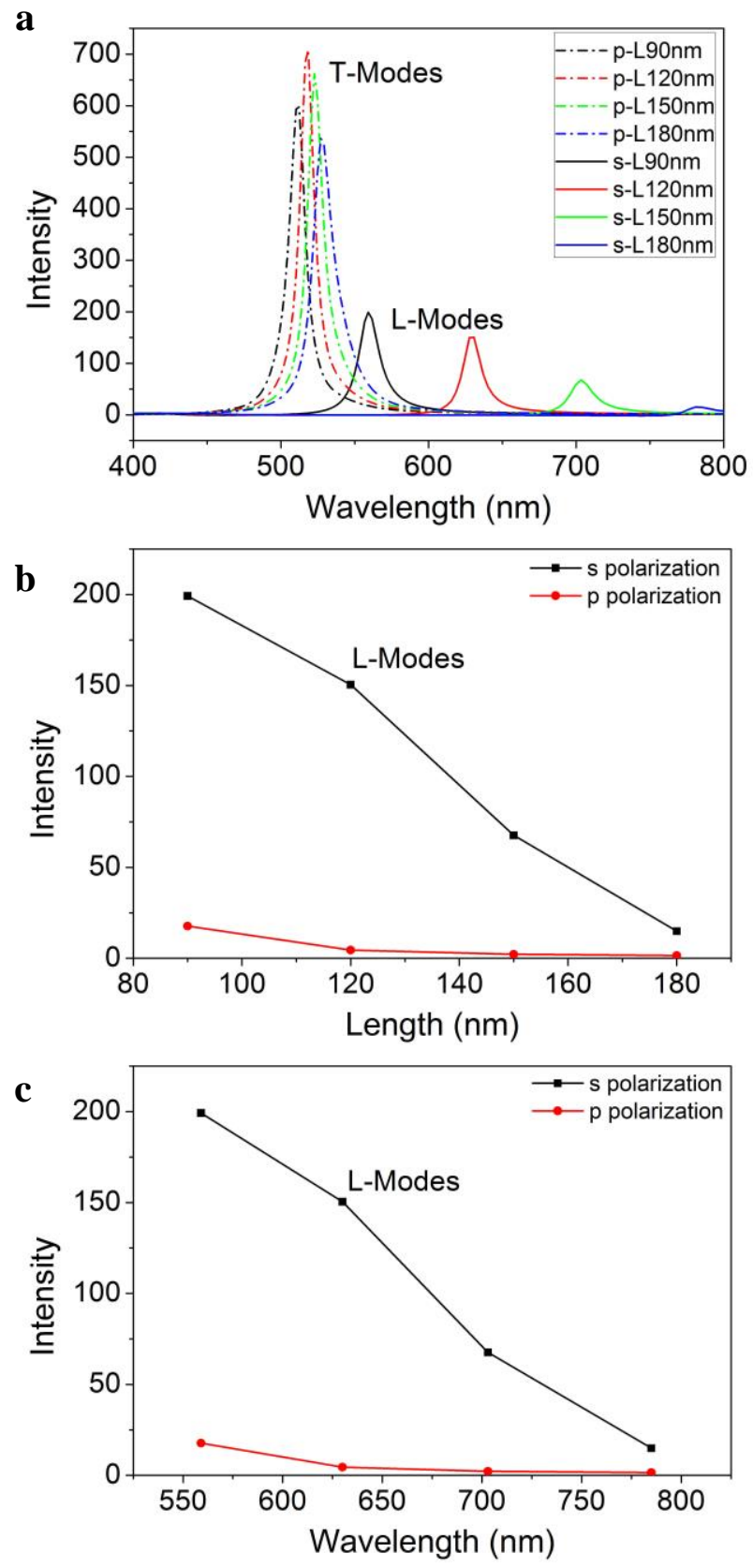

Figure 5. The spectra of localized surface plasmons of the silver nanorod metamaterials with the nanorod lengths of $90 \mathrm{~nm}, 120 \mathrm{~nm}$, $150 \mathrm{~nm}$ and $180 \mathrm{~nm}$ for the same nanorod diameter of $60 \mathrm{~nm}$. (a) T-Modes excited by the $p$-polarized wave (dash dot curves) and LModes excited by the s- polarized wave (solid curves); (b) L-Modes excited by the $s$ - polarized wave for the different lengths of the nanorods (black curve), the resonance at the L-Mode wavelengths by the $p$-polarized wave for the different lengths (red curve); (c) LModes excited by the $s$ - polarized wave for the different wavelengths corresponding to the different lengths of the nanorods (black curve), the out of resonance at the L-Mode wavelengths by the $p$ - polarized wave for the different wavelengths (red curve). 


\section{CONCLUSION}

The transversal and longitudinal resonance modes of the plasmonic nanorod metamaterials are investigated. It is found that the transversal mode (T-Mode) and the longitudinal mode (L-Mode) can be excited by $p$ - and $s$ - polarized waves, respectively, and the resonance intensity of the T-Modes excited by the $p$-polarized wave is obviously larger than the intensity for the $s$ - polarized wave at the resonance wavelengths of the T-Modes, and the resonance intensity of the LModes excited by the $s$ - polarized wave is clearly larger than the intensity for the $p$ - polarized wave at the resonance wavelengths of the L-Modes. As a result, the silver nanorod metamaterials attenuate the incident light at the resonance wavelength of one resonance mode (e.g., T-Mode or L-Mode) for one polarized wave (e.g., $p$ - polarization or $s$ polarization), while, they nearly completely transmit the incident light at the same wavelength for the other polarized wave ( $s$ - polarization or $p$ - polarization), which performs an obvious polarization behaviors. Furthermore, the electric field distributions of the plasmonic nanorods based on FDTD simulation presents the polarization behavior in the near field regions for the different resonance modes at the same wavelengths. On the other hand, the wavelength of the polarizers in the visible spectral range can be tuned by the diameter and length of the silver nanorods, higher extinction ratios and lower insertion losses can be obtained by the different resonance modes for the different polarizations. The polarizers can be used for the integration of nanophotonic devices.

\section{ACKNOWLEDGEMENTS}

This work was supported by National Natural Science Foundation of China $(51771186,11574070,10804112,11874012)$; The Fundamental Research Funds for the Central Universities (PA2018GDQT0006, JZ2017HGTB0187, PA2017GDQT0024, JZ2017HGBZ0942); The Seventh Framework Program of the European Union for Research through Marie Curie Actions-International Incoming Fellowships (623473, 913473); Program 973 (2015GB103003); The Project of State Key Laboratory of Environment-friendly Energy Materials, Southwest University of Science and Technology (17FKSY0109); China Postdoctoral Science Foundation (2015M571918, 2017T100442); The European Union's Horizon 2020 research and innovation program under the Marie Skłodowska-Curie grant agreement (744817).

\section{REFERENCES}

1. W. Zhou, M. Dridi, J. Y. Suh, C. H. Kim, D. T. Co, M. R. Wasielewski, G. C. Schatz, and T. W. Odom, "Lasing action in strongly coupled plasmonic nanocavity arrays," Nat. Nanotechnol. 8, 506-511 (2013).

2. F. van Beijnum, P. J. van Veldhoven, E. J. Geluk, M. J. A. de Dood, G. W. t Hooft, and M. P. van Exter, "Surface plasmon lasing observed in metal hole arrays," Phys. Rev. Lett. 110, 206802 (2013).

3. C. Zhang, Y. Lu, Y. Ni, M. Li, L. Mao, C. Liu, D. Zhang, H. Ming, and P. Wang, "Plasmonic lasing of nanocavity embedding in metallic nanoantenna array," Nano Lett. 15, 1382-1387 (2015).

4. A. V. Kabashin, P. Evans, S. Pastkovsky, W. Hendren, G. A. Wurtz, R. Atkinson, R. Pollard, V. A. Podolskiy, and A. V. Zayats, "Plasmonic nanorod metamaterials for biosensing," Nat. Mater. 8, 867-871 (2009).

5. C. R. Simovski, P. A. Belov, A. V. Atrashchenko, and Y. S. Kivshar, "Wire metamaterials: physics and applications," Adv. Mater. 24, 4229-4248 (2012).

6. L. Vigderman, B. P. Khanal, and E. R. Zubarev, "Functional gold nanorods: synthesis, self-assembly, and sensing applications," Adv. Mater. 24, 4811-4841 (2012).

7. R. Zhang, Y. Zhang, Z. C. Dong, S. Jiang, C. Zhang, L. G. Chen, L. Zhang, Y. Liao, J. Aizpurua, Y. Luo, J. L. Yang, and J. G. Hou, "Chemical mapping of a single molecule by plasmon-enhanced Raman scattering," Nature 498, 82-86 (2013).

8. H. Ditlbacher, A. Hohenau, D. Wagner, U. Kreibig, M. Rogers, F. Hofer, F. R. Aussenegg, and J. R. Krenn, "Silver nanowires as surface plasmon resonators," Phys. Rev. Lett. 95, 257403 (2005).

9. N. P. de Leon, B. J. Shields, C. L. Yu, D. E. Englund, A. V. Akimov, M. D. Lukin, and H. Park, "Tailoring light-matter interaction with a nanoscale plasmon resonator," Phys. Rev. Lett. 108, 226803 (2012).

10. L. Novotny, "Effective wavelength scaling for optical antennas," Phys. Rev. Lett. 98, 266802 (2007).

11. E. Cubukcu, and F. Capasso, "Optical nanorod antennas as dispersive one-dimensional Fabry-Peacuterot resonators for surface plasmons," Appl. Phys. Lett. 95, 201101 (2009).

12. J. Zhang, and L. Zhang, "Nanostructures for surface plasmons," Adv. Opt. Photon. 4, 157-321 (2012).

13. H. Song, J. Zhang, G. Fei, J. Wang, K. Jiang, P. Wang, Y. Lu, I. Iorsh, W. Xu, J. Jia, L. Zhang, Y. S. Kivshar, and L. Zhang, "Near-field coupling and resonant cavity modes in plasmonic nanorod metamaterials," Nanotechnology 27, 415708 (2016).

14. B. Song, Y. Yao, R. E. Groenewald, Y. Wang, H. Liu, Y. Wang, Y. Li, F. Liu, S. B. Cronin, A. M. Schwartzberg, S. Cabrini, S. Haas, and W. Wu, "Probing gap plasmons down to subnanometer scales using collapsible nanofingers," Acs Nano 11, 5836-5843 (2017).

15. J. Wang, C. Zhang, J. Zhang, H. Song, P. Wang, Y. Lu, G. Fei, W. Xu, W. Xu, L. Zhang, Y. S. Kivshar, and L. Zhang, "Hybrid plasmonic cavity modes in arrays of gold nanotubes," Adv. Opt. Mater. 5, 1600731 (2017). 